\title{
Prevalence of aflatoxin B1 in liver biopsies of proven hepatocellular carcinoma in India determined by an in-house immunoperoxidase test
}

\begin{abstract}
Correspondence
K. G. Murugavel murugavel@yrgcare.org
\end{abstract}

Received 4 January 2007

Accepted 29 June 2007

\author{
K. G. Murugavel, ${ }^{1}$ P. P. Naranatt, ${ }^{1}$ E. M. Shankar, ${ }^{1}$ S. Mathews, ${ }^{2}$ \\ K. Raghuram, ${ }^{2}$ P. Rajasambandam, ${ }^{2}$ V. Jayanthi, ${ }^{3}$ R. Surendran, ${ }^{3}$ \\ A. Murali, ${ }^{3}$ U. Srinivas, ${ }^{3}$ K. R. Palaniswamy, ${ }^{3}$ D. Srikumari ${ }^{4}$ \\ and S. P. Thyagarajan ${ }^{1}$
}
${ }^{1}$ Department of Microbiology, Faculty of Medicine, Dr ALM PG Institute of Basic Medical Sciences, University of Madras, Taramani Campus, Chennai 600 113, India
${ }^{2}$ Medical Gastroenterology Unit of the Government General Hospital, Chennai 600001 , India
${ }^{3}$ Medical and Surgical Gastroenterology Unit of the Government Stanley Medical College and Hospital, Chennai 600 001, India
${ }^{4}$ Government Kilpauk Medical College and Hospital, Chennai 600 029, India

\section{INTRODUCTION}

Hepatocellular carcinoma (HCC) is the fourth leading cause of cancer-related death worldwide (Parkin et al., 1997; Dhir \& Mohandas, 1998; Shibuya et al., 2002; ElSerag, 2004; Block et al., 2003). In the year 2000, it was projected that there would be 430000 deaths from HCC throughout the world. The prevalence of HCC in autopsies in India is low, and varies between 0.2 and $1.9 \%$ (Dhir \& Mohandas, 1998). Age-adjusted incidence of HCC in rural and urban Indian populations is low compared to that in

Abbreviations: AFB1, aflatoxin B1; DAB, 3'3'-diaminobenzidine tetrahydrochloride; HBsAg, hepatitis B surface antigen; HCC, hepatocellular carcinoma.
China, Japan and other Southeast Asian countries (Parkin et al., 1997; Dhir \& Mohandas, 1998). The risk of HCC is greatly increased in chronic viral carriers exposed to other recognized risk factors, including exposure to aflatoxin B1 (AFB1) (World Health Organization, 1998; Marrero et al., 2005).

AFB1, a secondary metabolite produced by Aspergillus flavus and Aspergillus parasiticus, occurs in tropical and subtropical regions of the world in which grains such as rice are stored under tropical conditions (Busby \& Wogan, 1984). In the months after the monsoon in Southeast Asia, stored grains reportedly contain high levels of AFB1. The grains stored under such conditions increase the risk of development of HCC, as AFB1 is a potent hepatocarcinogen. 
Based on various epidemiological data, a causative role for aflatoxins in hepatocarcinogenesis in humans has been strongly suggested. According to one study, the degree of food contamination by aflatoxins and HCC incidence are closely and significantly correlated (Van Rensburg et al., 1985). Another study has shown that the effects of exposure to aflatoxin and hepatitis B virus (HBV) infection are significantly associated with the risk of death due to liver cancer (Hoseyni, 1992).

Carcinogenic activity of aflatoxin is attributed to covalent adduct formation by metabolically activated reactive intermediates with hepatocyte DNA, which could lead to mutations in the host genome (Lin et al., 1977). Mutations of the p53 gene on chromosome 17 have been frequently found in primary liver cancer (Hsu et al., 1991; Bressac et al., 1991; Laurent-Puig et al., 2001). This hotspot mutation was originally described in HCC from regions with high levels of dietary aflatoxins, and is considered a hallmark of AFB1. The molecular and cellular mechanisms underlying the carcinogenic effects of aflatoxin have also been investigated on numerous occasions in rodent models. N-ras and c-Ki-ras oncogenes have been characterized in AFB1-induced HCC in rats (McMahon et al., 1990).

Until recently, the situation in humans has been less clear because of the difficulty in assessing the degree of exposure. This has, to some extent, been overcome by the development of biomarkers of exposure, which detect aflatoxin metabolites and DNA adducts in urine and blood (Ross et al., 1992). However, it is difficult to assess the past intake of aflatoxin in urine and blood, which provides only an indirect measure of intake in the immediate past. The other method of detecting AFB1 intake in the past was immunocytochemical localization of aflatoxin in individual cells.

Epidemiological surveys have revealed a strong statistical correlation between aflatoxin ingestion and incidence of HCC in several areas of the world. Data on the epidemiology of AFB1 in human HCC have never been reported from liver biopsies from parts of southern India; therefore, we developed a novel immunoperoxidase assay in Chennai, India, to measure AFB1 in tissue and serum specimens collected from clinically proven cases of HCC.

\section{METHODS}

Clinical samples. From 1994 to 1998, we investigated 1012 patients with clinically diagnosed chronic liver disease who attended the various medical and surgical gastroenterology units of government hospitals in Chennai city. The patients were analysed, based on inclusion/exclusion criteria, using a statistical stratified proforma. Inclusion in the present study was based on clinical diagnosis. Patients who showed an abdominal mass on ultrasound and/or elevated $\alpha$-fetoprotein levels were enrolled. The study cases were classified as chronic persistent hepatitis $(n=46)$, chronic active hepatitis, cirrhosis and HCC based on the histopathological reports of liver biopsy. Each subject provided written informed consent to conduct an interview, collect a blood specimen, undergo liver biopsy and perform serological assays. All the patients were interviewed in person with the use of a structured questionnaire. The subjects were questioned about socio-demographic characteristics, diet, cigarette smoking, consumption of alcohol, betel-nut chewing, medical and surgical history, and any family history of HCC or liver cirrhosis.

Of these 1012 cases, 742 had chronic liver disease and 170 had HCC. Of the 170 histopathologically proven HCC cases screened, we tested 31 liver biopsies and 7 liver-resection specimens for AFB1. In addition, we collected liver-resection specimens from seven HCC cases. Serum specimens from all the HCC cases were screened for AFB1 by an in-house ELISA.

A $10 \mathrm{ml}$ specimen of blood was collected using a disposable vacuum syringe, and separated sera were kept in a freezer $\left(-70{ }^{\circ} \mathrm{C}\right)$ until they were assayed. Liver biopsies were preserved in $10 \%$ formalin.

Detection of hepatitis markers by ELISA. The serum specimens were tested for hepatitis B surface antigen (HBsAg), hepatitis B e antigen, antibody to hepatitis B e antigen, IgM antibody to hepatitis B core antigen, hepatitis delta antigen and antibody to hepatitis delta virus (Wellcozyme; Murex Diagnostics) by ELISA, with the use of commercially available kits according to the manufacturer's instructions. Anti-hepatitis C virus (HCV) status was tested using the RIBA 3.0 SIA in vitro qualitative enzyme immunoassay. From the formalinfixed liver biopsies/resected livers, sections were made for AFB1 detection by an in-house immunoperoxidase method. In addition, the tumour and non-tumour areas of the same HCC cases were examined for AFB1.

Detection of AFB1 by an in-house ELISA and in-house immunoperoxidase test. AFB1 detection in serum specimens was performed using an in-house ELISA developed as per standard procedures (Wilkinson et al., 1988) with slight modifications, in which the peroxidase-conjugated anti-AFB1 was used at a concentration of $1: 1000$. All the chemicals and reagents used in the investigation were of analytical grade and were procured from Sigma. The test was sensitive enough to detect up to $\sim 1 \mathrm{ng} \mathrm{ml}^{-1}$ of AFB1 in serum. The immunoperoxidase test was as per a standardized inhouse assay to localize AFB1 in liver specimens. Briefly, the paraffin sections were dewaxed in xylene and rehydrated through descending grades of alcohol. The slides with sections were washed in $0.5 \mathrm{M}$ glycine in PBS ( $\mathrm{pH}$ 7.2) to inhibit/remove fixing agents such as aldehydes, which can cause non-specific binding of immunoperoxidase or reduced $3^{\prime}, 3^{\prime}$-diaminobenzidine tetrahydrochloride (DAB). Endogenous peroxidase activity was blocked with a fresh $0.3-0.5 \%$ solution of $\mathrm{H}_{2} \mathrm{O}_{2}$ in absolute methanol for $30 \mathrm{~min}$. The slides were then washed in Tris buffer for $5 \mathrm{~min}$ and incubated with a few drops of $3 \%$ normal goat serum for $20-30 \mathrm{~min}$. Four to six drops of peroxidase-conjugated anti-AFB1 $(1: 1000-1: 750)$ were added to the slides and incubated for $30 \mathrm{~min}$. Anti-AFB1 was raised in rabbits, showed high affinity to AFB1, and did not cross-react with aflatoxin G2a, B2a or M1. The slides were rinsed with buffer. Substrate was prepared by dissolving $5 \mathrm{mg} \mathrm{DAB}$ in $10 \mathrm{ml} 0.05 \mathrm{M}$ Tris/ $\mathrm{HCl}$ (pH 7.6) and adding $20 \mu \mathrm{l} 30 \% \mathrm{H}_{2} \mathrm{O}_{2}$. Substrate solution (5-10 drops) was placed on the slides and incubated for 5-8 $\mathrm{min}$ at room temperature in the dark. The sections were rinsed with tap water and counterstained with Mayer's haematoxylin. After dehydrating the sections in graded alcohols, they were cleared with xylene before mounting in DPX (a mixture of distyrene, a plasticizer and xylene). The slides were observed under a light microscope $(\times 400$ magnification) for the presence of dark-brown reaction products, formed by the reaction between the anti-AFB1 and DAB (Fig. 1).

Statistical analysis. Statistical analysis was done using Epi Info statistical software (http://www-unix.oit.umass.edu/ statdata/software/ handouts/epiinfo.html). $P \leqslant 0.05$ was considered to show significance. 

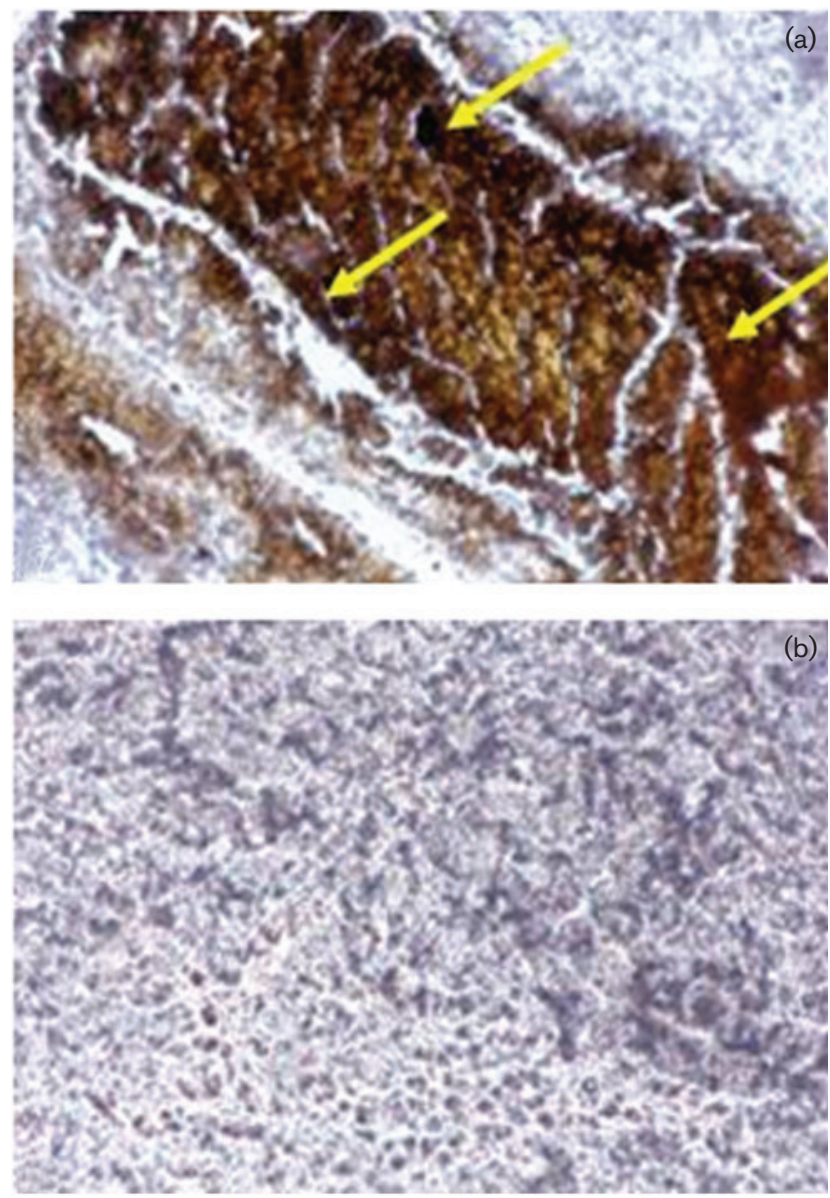

Fig. 1. In-house immunoperoxidase assay shows localization of AFB1 in liver biopsy sections from patients with HCC, counterstained with Mayer's haematoxylin. (a) Dark-brown coloration (yellow arrows) formed by the reaction between anti-AFB1 and DAB. (b) Absence of dark-brown coloration in AFB1-negative sections. Magnification $\times 400$.

\section{RESULTS}

Liver tissue biopsies from $31 \mathrm{HCC}$ and 15 cirrhosis cases, aged $>18$ years, were investigated for AFB1. The male:female ratio for HCC in the present study was $6.4: 1$. The mean age for HCC was 62.7 years for males and 46 years for females. The clinical symptomatology revealed an abdominal mass in $64.1 \%$ of cases, while anorexia and abdominal pain were seen in 43.5 and $35.9 \%$, respectively. Other common symptoms were pedal oedema $(27.6 \%)$, ascites $(24.7 \%)$, weight loss $(21.2 \%)$, fever $(12.9 \%)$ and haematemesis $(8.8 \%)$. The mean age of HCC patients positive for aflatoxin alone was 53.9 years, compared with 61.1 years for those that were positive for both HBV and AFB1.

The biopsies from HCC and cirrhosis cases were analysed for in situ positivity for AFB1 by the immunoperoxidase staining method. When the liver biopsies were investigated for AFB1 in liver sections, 18 (58.1\%) cases with HCC revealed AFB1, but all cirrhosis cases were negative for AFB1. The mean age of the AFB1-positive patients was 55 years, whereas the mean age of the $20(61.9 \%)$ AFB1negative HCC patients was 50 years. The male to female ratio was $8.5: 1$ in the AFB1-positive patients and $3.7: 1$ in the AFB1-negative HCC patients. Microscopic examination of the sections revealed dark-brown coloration due to the reaction between anti-AFB1 and DAB in the HCC cases (Fig. 1). Furthermore, while $11(68.8 \%)$ liver biopsies from non-B, non-C hepatitis cases with HCC were positive for AFB1, six (46.1\%) HBsAg-positive subjects were also positive for AFB1 deposits. Out of the two HBV/HCV coinfected patients, one was positive for AFB1 (Table 1). In spite of positive AFB1 immunostaining in HCC cases, all serum specimens, from both the HCC and control groups, were AFB1-negative with serum ELISA. In addition, seven tumour-resection samples were studied. Six of these (85.7\%) were positive for AFB1, and AFB1 staining was associated with the tumour tissue. Interestingly, only one case revealed AFB1 in the non-tumour area of the resection material. Thus AFB1 staining was significantly associated with tumour tissue $(P=0.03)$.

\section{DISCUSSION}

Hepatocarcinogenesis is a multistage, multifactorial process, involving viral, chemical and several other factors, including aflatoxins, alcohol and tobacco consumption, and familial tendencies (Chen et al., 1992; Wang et al., 2001; Williams et al., 2004). Nevertheless, special emphasis has been placed on aflatoxins, because of the frequency with which they occur as food contaminants, together with their potency as liver carcinogens in numerous animal species.

The biomarker-based, prospective Shanghai Cohort Study results have been subsequently confirmed by other independent cohorts, and unequivocally demonstrate a strong association between baseline levels of urinary AFB metabolites, especially the major AFB-DNA adduct, and HCC risk in study subjects (Chen et al., 1992; Wang et al., 2001; Williams et al., 2004). In addition, the International

Table 1. Tissue positivity for AFB1 in $\mathrm{HCC}$ as per viral aetiology

\begin{tabular}{|lcc|}
\hline \multirow{2}{*}{ HCC group } & \multicolumn{2}{c|}{ Tissue AFB1 $(\boldsymbol{n}=\mathbf{3 1})$} \\
\cline { 2 - 3 } & Numbers & Positivity $^{\star}$ \\
\hline HBV & 13 & $6(46.1)$ \\
HCV & ND & ND $(0)$ \\
HBV + HCV & 2 & $1(50)$ \\
Non-B non-C & 16 & $11(68.8)$ \\
\hline
\end{tabular}

ND, Not done.

${ }^{\star}$ Numbers in parentheses indicate percentage. 
Agency for Research on Cancer (IARC) in Lyon, France, has classified aflatoxin as a group 1 human carcinogen (IARC, 1993). Several ecological studies have also shown a significant association between dietary aflatoxin exposure and hepatocarcinogenesis (Peers et al., 1987; Yeh et al., 1989). However, results refuting the association have also been reported on several occasions (Lam et al., 1982; Lu et al., 1988; Campbell et al., 1990). This inconsistency may be due to the difficulty of accurate assessment of aflatoxin exposure at the population or individual level. Most of the methods used for the quantification of aflatoxins require skilled manipulation, sophisticated and expensive equipment, and an elaborate laboratory set-up. Nevertheless, specific antibodies against AFB1 metabolites are useful alternatives to these approaches, and might be used to discern the intracellular fate of AFB1 in the liver and other organs at the ultrastructural level.

However, in India and many other developing tropical countries, meaningful strategies for implementing legislation to control AFB1 contamination in food are limited by the non-availability of simple, rapid, cost-effective and reliable ELISA methods to detect AFB1 in serum. Therefore, we developed a novel in-house immunoperoxidase technique to detect AFB1 in liver biopsies in Chennai, India.

There have been no reports from India in relation to the degree of contamination of food for human consumption by aflatoxin. An outbreak of toxic hepatitis with a high mortality among the exposed villagers and animals in India has been reported previously (Tandon et al., 1977). Our study ruled out AFB1 and AFB2 in all the serum samples from HCC and cirrhosis cases by ELISA. Even though the test worked satisfactorily with the positive controls, certain limitations were observed with the kit, i.e. its detection limit was only $1 \mathrm{ng} \mathrm{ml}^{-1}$. Furthermore, none of the test serum samples were fresh, but were deep-frozen for varying periods of time.

The other method of choice to demonstrate past intake of AFB in HCC cases is to look for localization of AFB1 in liver sections (Chen et al., 1992). In a Kenyan study that investigated liver biopsies and blood and urine specimens for AFB1 from HCC and cirrhosis patients, $63 \%$ of HCC cases had positive liver specimens, whereas blood samples were negative (De Vries \& Lamplugh, 1989). However, all the three cirrhosis cases showed AFB1 in blood and urine, but only one liver specimen showed the localization of aflatoxin. Our in-house immunoperoxidase test carried out on liver biopsies of HCC detected AFB1 in $58 \%$ of cases. Nonetheless, no cirrhosis case was positive for the aflatoxin. This suggests that these patients had a possible history of AFB1-contaminated foodstuffs, which has acted either as a primary or additional risk factor for HCC. To the best of our knowledge, no Indian report is available in the literature on the role of aflatoxin in HCC.

The evidence from this study supports the hypothesis that dietary aflatoxin, rather than acting as a primary carcinogen, as it seems to do in animals, suppresses cellmediated immunity. This effect on the immune system will allow HBV, highly endemic in certain populations, to maintain itself more easily in the liver, to produce more chronic infection and cirrhosis, and, in the long term, to cause a high incidence of HCC. An analysis of our results along these lines showed the aflatoxin level to be high in non-carriers of $\mathrm{HBV}$, which is indicative of the independent role played by AFB1 in hepatocarcinogenesis, with $68.7 \%$ of the non- $\mathrm{B}$ non- $\mathrm{C}$ hepatitis cases having tissue localization of AFB1.

AFB1 detection in the present study seemed to be significantly higher among men (64\%) than among women $(33.3 \%)$. This observation differs from that of others, who have shown a slightly lower detection rate in men than in women (Chen et al., 1992). Similarly, our observation also differs from others in the age group predilection of aflatoxin-induced HCC in Taiwan. Chen et al. (1992) have shown that younger patients had a significantly higher rate of AFB1 (83\%) than older patients (58\%).

The overall analysis of aflatoxin positivity with or without viral hepatitis positivity reveals the following: (1) aflatoxins have an independent role in bringing about hepatocarcinogenesis, as revealed by their significant presence in the liver of non-viral HCC cases also; and (2) the concomitant presence of AFB1 along with HBV in $46.1 \%$ of cases studied is an additional risk factor to which populations from developing countries are exposed, and may play an added aggravating role in hepatocarcinogenesis. Our study, with six (46.1\%) HBsAg-positive cases with HCC, is in agreement with a previous Indian study in finding that HBsAg positivity in patients with HCC varies from 36 to $74 \%$, with a mean of $47 \%$ (Dhir \& Mohandas, 1998).

The conventional methods for aflatoxin detection, i.e. GLC and HPLC, are cumbersome and require expensive equipment. Hence we developed an in-house ELISA for the detection of aflatoxin (AFB1) in blood specimens from HCC and non-HCC patients with cirrhosis. A major methodological problem limiting our ability to study the role of aflatoxin in HCC is the absence of markers of past exposure in patients. The other alternative for assessing the past intake of aflatoxin is to examine the liver tissue. This immunoperoxidase method needs less equipment than other techniques (e.g. immunofluorescence assays). In addition, implementation of this method for the detection of AFB1 might be possible only upon proper validation and thorough evaluation of the technique for use in clinical studies. This approach may be suggested as a rapid, reliable and cost-effective substitute for other tests to detect AFB1 in less-equipped laboratory settings after thorough evaluation and validation.

In conclusion, we reported that, using an in-house immunoperoxidase staining test, $58.1 \%$ of HCC cases showed AFB1 in liver biopsies, but all cirrhosis cases were negative for AFB1. Furthermore, $68.8 \%$ of HBsAg-negative 
HCC cases and $46.1 \%$ of HBsAg-positive patients were positive for AFB1 in liver biopsy, which proves that aflatoxins have a significant association with HCC in this part of the tropical subcontinent.

\section{REFERENCES}

Block, T. M., Mehta, A. S., Fimmel, C. J. \& Jordan, R. (2003). Molecular viral oncology of hepatocellular carcinoma. Oncogene 22, 5093-5107.

Bressac, B., Kew, M., Wands, J. \& Ozturk, M. (1991). Selective G to T mutations of p53 gene in hepatocellular carcinoma from southern Africa. Nature 350, 429-431.

Busby, W. F., Jr \& Wogan, G. N. (1984). Aflatoxins. In Chemical Carcinogens, vol. 2, 2nd edn, pp. 945-1136. Edited by C. E. Searle. Washington, DC: American Chemical Society.

Campbell, T. C., Chen, J., Liu, C., Li, J. \& Parpia, B. (1990). Nonassociation of aflatoxin with primary liver cancer in a crosssectional ecological survey in the People's Republic of China. Cancer Res 50, 6882-6893.

Chen, C. J., Zhang, Y. J., Lu, S. N. \& Santell, R. M. (1992). Aflatoxin B1 DNA adducts in smeared tumor tissue from patients with hepatocellular carcinoma. Hepatology 16, 1150-1155.

De Vries, H. R. \& Lamplugh, S. M. (1989). Aflatoxins in liver biopsies from Kenya. Trop Geogr Med 41, 26-30.

Dhir, V. \& Mohandas, K. M. (1998). Epidemiology of digestive cancer in India. III. Liver. Indian J Gastroenterol 17, 100-103.

El-Serag, H. B. (2004). Hepatocellular carcinoma: recent trends in the United States. Gastroenterology 127, S27-S34.

Hoseyni, M. S. (1992). Risk assessment for aflatoxin. III. Modeling the relative risk of hepatocellular cancer. Risk Anal 12, 123-128.

Hsu, I. C., Metcalf, R. A., Sun, T., Welsh, J. A., Wang, N. J. \& Harris, C. C. (1991). Mutational hotspot in the p53 gene in human hepatocellular carcinomas. Nature 350, 427-428.

IARC (1993). Aflatoxins. IARC Monogr Eval Carcinog Risks Hum 56, 245-395.

Lam, K. C., Yu, M. C., Leung, J. W. C. \& Henderson, B. E. (1982). Hepatitis B virus and cigarette smoking: risk factors for hepatocellular carcinoma in Hong Kong. Cancer Res 42, 5246-5248.

Laurent-Puig, P., Legoix, P., Bluteau, O., Belghiti, J., Franco, D. Binot, F., Monges, G., Thomas, G., Bioulac-Sage, P. \& ZucmanRossi, J. (2001). Genetic alterations associated with hepatocellular carcinomas define distinct pathways of hepatocarcinogenesis. Gastroenterology 120, 1763-1773.

Lin, J. K., Mille, J. A. \& Miller, E. C. (1977). 2,3-Dihydro-2-(guan-7-yl)3-hydroxy-aflatoxin B1, a major acid hydrolysis product of aflatoxin B1-DNA or ribosomal RNA adducts formed in hepatic microsomemediated reactions in rat liver in vivo. Cancer Res 37, 4430-4438.
Lu, S. N., Lin, T. M., Chen, C. J., Chen, J. S., Liaw, Y. F., Chang, W. Y. \& Hsu, S. T. (1988). A case-control study of primary hepatocellular carcinoma in Taiwan. Cancer 62, 2051-2055.

Marrero, J. A., Fontana, R. J., Fu, S., Conjeevaram, H. S., Su, G. L. \& Lok, A. S. (2005). Alcohol, tobacco and obesity are synergistic risk factors for hepatocellular carcinoma. J Hepatol 42, 218-224.

McMahon, G., Davis, E. F., Huber, L. J., Kim, Y. \& Wogan, G. N. (1990). Characterization of c-Ki-ras and $\mathrm{N}$-ras oncogenes in aflatoxin B1induced rat liver tumors. Proc Natl Acad Sci U S A 87, 1104-1108.

Parkin, D. M., Whelan, S. L., Ferlay, J., Raymond, L. \& Young, J. (1997). Cancer Incidence in Five Continents, vol. VII. Lyon: International Agency for Research on Cancer.

Peers, F., Bosch, X., Kaldor, J., Linsell, A. \& Pluijmen, M. (1987). Aflatoxin exposure, hepatitis B virus infection and liver cancer in Swaziland. Int J Cancer 39, 545-553.

Ross, R. K., Yuan, J. M., Yu, M. C., Wogan, G. N., Qian, G. S., Tu, J. T., Groopman, J. D., Gao, Y. T. \& Henderson, B. E. (1992). Urinary aflatoxin biomarkers and risk of hepatocellular carcinoma. Lancet 339, 943-946.

Shibuya, K., Mathers, C. D., Boschi-Pinto, C., Lopez, A. D. \& Murray, C. J. (2002). Global and regional estimates of cancer mortality and incidence by site: II. Results for the global burden of disease. BMC Cancer 2, 37.

Tandon, B. N., Krishnamurthy, L., Koshy, A., Tandon, H. D., Ramalingaswami, V., Bhandari, J. R., Mathur, M. M. \& Mathur, P. D. (1977). Study of an epidemic of jaundice, presumably due to toxic hepatitis in Northwest India. Gastroenterology 72, 488-494.

Van Rensburg, S. J., Cook-Mozaffari, P., Van Schalkwyk, D. J., Van Der Watt, J. J., Vincent, T. J. \& Purchase, I. F. (1985). Hepatocellular carcinoma and dietary aflatoxin in Mozambique and Transkei. $\mathrm{Br} J$ Cancer 51, 713-726.

Wang, J. S., Huang, T., Su, J., Liang, F., Wei, Z., Liang, Y., Luo, H., Kuang, S.-Y., Qian, G.-S. \& other authors (2001). Hepatocellular carcinoma and aflatoxin exposure in Zhuqing Village, Fusui County, People's Republic of China. Cancer Epidemiol Biomarkers Prev 10, 143-146.

World Health Organization (1998). Food additives series 40. In Safety Evaluations of Certain Food Additives and Contaminants. Aflatoxins, pp. 359-468. Geneva: World Health Organization.

Wilkinson, A. P., Denning, D. W. \& Morgan, M. R. A. (1988). Analysis of UK sera for aflatoxin by enzyme-linked immunosorbent assay. Hum Toxicol 7, 353-356.

Williams, J. H., Phillips, T. D., Jolly, P. E., Stiles, J. K., Jolly, C. M. \& Aggarwal, D. (2004). Human aflatoxicosis in developing countries: a review of toxicology, exposure, potential health consequences, and interventions. Am J Clin Nutr 80, 1106-1122.

Yeh, F. S., Yu, M. C., Mo, C. C., Luo, S., Tong, M. J. \& Henderson, B. E. (1989). B virus, aflatoxins and hepatocellular carcinoma in southern Guangxi, China. Cancer Res 49, 2506-2509. 